\title{
Fractional Variational Iteration Method versus Adomian's Decomposition Method in Some Fractional Partial Differential Equations
}

\author{
Junqiang Song, ${ }^{1}$ Fukang Yin, ${ }^{1}$ Xiaoqun Cao, ${ }^{1}$ and Fengshun $\mathrm{Lu}^{2}$ \\ ${ }^{1}$ College of Computer, National University of Defense Technology, Changsha, Hunan 410073, China \\ ${ }^{2}$ China Aerodynamics Research and Development Center, Mianyang, Sichuan 621000, China \\ Correspondence should be addressed to Fukang Yin; yinfukang@nudt.edu.cn
}

Received 17 September 2012; Revised 28 December 2012; Accepted 31 December 2012

Academic Editor: Zhongxiao Jia

Copyright (C) 2013 Junqiang Song et al. This is an open access article distributed under the Creative Commons Attribution License, which permits unrestricted use, distribution, and reproduction in any medium, provided the original work is properly cited.

A comparative study is presented about the Adomian's decomposition method (ADM), variational iteration method (VIM), and fractional variational iteration method (FVIM) in dealing with fractional partial differential equations (FPDEs). The study outlines the significant features of the ADM and FVIM methods. It is found that FVIM is identical to ADM in certain scenarios. Numerical results from three examples demonstrate that FVIM has similar efficiency, convenience, and accuracy like ADM. Moreover, the approximate series are also part of the exact solution while not requiring the evaluation of the Adomian's polynomials.

\section{Introduction}

Fractional differential equations (FDEs), as a generalization of ordinary differential equations to an arbitrary (noninteger) order, have been proved to be a valuable tool in modelling many phenomena in the fields of physics, chemistry, engineering, aerodynamics, electrodynamics of complex medium, polymer rheology, and so forth [1-9]. The reasons are that fractional derivatives provide an excellent instrument for description of memory and hereditary properties of various materials and processes.

Considerable attention has been paid to developing accurate and efficient methods for solving fractional partial differential equations (FPDEs). Most of the nonlinear fractional differential equations do not have exact analytic solutions, so approximation and numerical techniques must be used. Recently, some approximate methods such as Adomian's decomposition method (ADM) [10-13], homotopy perturbation method (HPM) [14-16], variational iteration method (VIM) [17-22], homotopy analysis method (HAM) [23-26], fractional complex transform (FCT) [27-31], and wavelets method [32-34] have been given to find an analytical approximation to FDEs.
The variational iteration method (VIM), which was first proposed by He et al. [17-22] and has been shown to be very efficient for handling a wide class of physical problems. As early as 1998, the variational iteration method was shown to be an effective tool for factional calculus [35]; hereafter, the method has been routinely used to solve various fractional differential equations [11, 36-41] for many years. In order to improve the accuracy and efficiency of the VIM for factional calculus, a modification called fractional variational iteration method (FVIM) $[42,43]$ was proposed and some successes $[44,45]$ have been achieved. In the field of fractional differential equations, the main difference between VIM and FVIM is the evaluation of Lagrange multipliers: VIM usually get Lagrange multipliers by some approximations.

The three methods (i.e., VIM, FVIM, and ADM) are relatively new and effective approaches to find the approximate solution of PDEs, because they provide immediate and visible symbolic terms of analytic solutions, as well as numerical approximate solutions to both linear and nonlinear PDEs without linearization or discretization. The prior work [4649] has performed a comparative study of ADM and VIM and got two useful conclusions: on the one hand, ADM needs specific algorithms to evaluate the Adomian's polynomials, 
while VIM handles linear and nonlinear problems in a similar manner without any additional requirement or restriction; on the other hand, Adomian's decomposition method provides the components of the exact solution. However, it has to be validated whether these conclusions are also true for the scenario of FPDEs.

In this paper, we consider the following fractional initial value problem:

$$
D_{t}^{\alpha} u(x, t)+N[u(x, t)]+L[u(x, t)]=g(x, t), \quad t>0,
$$

where $L$ is a linear operator, $N$ is a nonlinear operator in $x, t$, and $D^{\alpha}$ is the modified Riemann-Liouville derivative of order $\alpha$, subject to the initial conditions

$$
\begin{array}{r}
u^{(k)}(x, 0)=c_{k}(x), \\
k=0,1,2, \cdots, m-1, m-1<\alpha \leq m .
\end{array}
$$

We will provide a comparative study of ADM and FVIM in dealing with the above FPDEs. The remainder of the paper is organized as follows. We begin by introducing some necessary definitions and mathematical preliminaries for the fractional calculus theory in Section 2. We present the VIM/FVIM method and the ADM in Sections 3 and 4, respectively. In Section 5, three examples are given to demonstrate our conclusions. Finally, a brief summary is presented.

\section{Preliminaries and Notations}

In this section, we describe some necessary definitions and mathematical preliminaries of the fractional calculus theory.

Definition 1. A real function $h(t), t>0$, is said to be in the space $C_{\mu}, \mu \in R$, if there exists a real number $p>\mu$, such that $h(t)=t^{p} h_{1}(t)$, where $h_{1}(t) \in C(0, \infty)$, and it is said to be in the space $C_{\mu}^{n}$ if and only if $h^{(n)} \in C_{\mu}, n \in N$.

Definition 2. Riemann-Liouville fractional integral operator $\left(J^{\alpha}\right)$ of order $\alpha \geq 0$, of a function $f \in C_{\mu}, \mu \geq-1$ is defined as

$$
\begin{gathered}
J^{\alpha} f(t)=\frac{1}{\Gamma(\alpha)} \int_{0}^{t}(t-\tau)^{\alpha-1} f(\tau) d \tau, \quad t>0, \\
J^{0} f(t)=f(t) .
\end{gathered}
$$

$\Gamma(\alpha)$ is the well-known gamma function. Some properties of the operator $J^{\alpha}$ can be found in $[4,8,9,50]$. We only recall the following ones:

$$
\begin{gathered}
J^{\alpha} J^{\beta} f(t)=J^{\alpha+\beta} f(t), \\
J^{\alpha} J^{\beta} f(t)=J^{\beta} J^{\alpha} f(t), \\
J^{\alpha} t^{\gamma}=\frac{\Gamma(\gamma+1)}{\Gamma(\alpha+\gamma+1)} t^{\alpha+\gamma},
\end{gathered}
$$

for $f \in C_{\mu}, \mu \geq-1, \alpha, \beta \geq 0$, and $\gamma>-1$.
The Riemann-Liouville derivative has certain disadvantages when trying to model real-world phenomena with FDEs. Therefore, we will introduce a modified fractional differential operator $D_{x}^{\alpha}$ proposed by Caputo [51].

Definition 3. The fractional derivative of $f(x)$ in the Caputo sense is defined as

$$
\begin{aligned}
& \left(D_{x}^{\alpha} f\right)(x) \\
& = \begin{cases}\frac{1}{\Gamma(m-\alpha)} \int_{0}^{x} \frac{f^{(m)}(\xi)}{(x-\xi)^{\alpha-m+1}} d \xi, & (\alpha>0, m-1<\alpha<m) \\
\frac{\partial^{m} f(x)}{\partial x^{m}}, & \alpha=m,\end{cases}
\end{aligned}
$$

where $f: R \rightarrow R, x \rightarrow f(x)$ denotes a continuous (but not necessarily differentiable) function.

Some useful formulas and results of modified RiemannLiouville derivative, which we need here, are listed as follows:

$$
\begin{gathered}
D_{x}^{\alpha} c=0, \quad \alpha>0, \quad c=\text { constant, } \\
D_{x}^{\alpha}[c f(x)]=c D_{x}^{\alpha} f(x), \quad \alpha>0, \quad c=\text { constant, } \\
D_{x}^{\alpha} x^{\beta}=\frac{\Gamma(1+\beta)}{\Gamma(1+\beta-\alpha)} x^{\beta-\alpha}, \quad \beta>\alpha>0, \\
D_{x}^{\alpha}[f(x) g(x)]=\left[D_{x}^{\alpha} f(x)\right] g(x)+f(x)\left[D_{x}^{\alpha} g(x)\right], \\
D_{x}^{\alpha}[f(x(t))]=f_{x}^{\prime}(x) x^{(\alpha)}(t) .
\end{gathered}
$$

Lemma 4. Let $n-1<\alpha \leq n, n \in N, t>0, h \in C_{\mu}^{n}, \mu \geq-1$. Then

$$
\left(J^{\alpha} D^{\alpha}\right) h(t)=h(t)-\sum_{k=0}^{n-1} h^{(k)}\left(0^{+}\right) \frac{t^{k}}{k !} .
$$

\section{VIM and FVIM}

3.1. Variational Iteration Method. In this section, the basic ideas of variational iteration method (VIM) are introduced. Here a description of the method (please refer to publications [17-19] for more details) is given to handle the general nonlinear problem as

$$
L(u)+N(u)=g(t)
$$

where $L$ is a linear operator, $N$ is a nonlinear operator, and $g(t)$ is a known analytic function.

According to He's variational iteration method [17-22], we can construct a correction functional as follows:

$$
\begin{aligned}
u_{n+1}(t)= & u_{n}(t) \\
& +\int_{0}^{t} \lambda(\tau)\left\{L\left(u_{n}(\tau)\right)+N\left(\widetilde{u}_{n}(\tau)\right)-g(\tau)\right\}, \quad n \geq 0,
\end{aligned}
$$

where $\lambda$ is a general Lagrange multiplier which can be optimally identified via variational theory and $\widetilde{u}_{n}$ is a restricted 
variation which means $\delta \tilde{u}_{n}=0$. Therefore, the Lagrange multiplier $\lambda$ should be first determined via integration by parts. The successive approximation $u_{n}(t)(n \geq 0)$ of the solution $u(t)$ will be readily obtained by using the obtained Lagrange multiplier and any selective function $u_{0}$. The zeroth approximation $u_{0}$ may be selected by any function that just meets, at least, the initial and boundary conditions. With $\lambda$ determined, then several approximations $u_{n}(t), n \geq 0$ follow immediately. Consequently, the exact solution may be obtained as

$$
u(t)=\lim _{n \rightarrow \infty} u_{n}(t)
$$

The VIM depends on the proper selection of the initial approximation $u_{0}(t)$. Finally, we approximate the solution of the initial value problem (1) by the $n$ th-order term $u_{n}(t)$. It has been validated that VIM is capable of effectively, easily, and accurately solving a large class of nonlinear problems.

3.2. Fractional Variational Iteration Method. We can construct a correction functional for (1) as follows:

$$
\begin{aligned}
& u_{k+1}(x, t) \\
& =u_{k}(x, t)+\int_{0}^{t} \lambda(t, \tau)\left(D_{\tau}^{\alpha} u_{k}(x, \tau)+N\left[\tilde{u}_{k}(x, \tau)\right]\right. \\
& \left.+L\left[\tilde{u}_{k}(x, \tau)\right]-g(x, \tau)\right) d \tau,
\end{aligned}
$$

where $\widetilde{u}(x, t)$ is a restricted variation.

Taking Laplace transform to both sides of (11) as

$$
\begin{gathered}
\bar{u}_{k+1}(x, t)=\bar{u}_{k}(x, t) \\
+\bar{L}\left[\int _ { 0 } ^ { t } \lambda ( t , \tau ) \left(D_{t}^{\alpha} u_{k}(x, \tau)+N\left[\tilde{u}_{k}(x, \tau)\right]\right.\right. \\
\left.\left.+L\left[\tilde{u}_{k}(x, \tau)\right]-g(x, \tau)\right) d \tau\right]
\end{gathered}
$$

where $\bar{u}_{k}(x, t)$ is Laplace transform of $u_{k}(x, t)$ with respect to $t$ and $\bar{L}$ is operator of Laplace transform.

By assuming that the Lagrange multiplier has the form as $\lambda(t, \tau)=\lambda(t-\tau)$, so that $\bar{L}\left[J_{\tau}^{\alpha} \lambda\left(D_{\tau}^{\alpha} u_{k}(x, \tau)+N\left[\tilde{u}_{k}(x, \tau)\right]+\right.\right.$ $\left.\left.L\left[\tilde{u}_{k}(x, \tau)\right]-g(x, \tau)\right)\right]$ is the convolution of the function $\lambda(t)$ and $D_{t}^{\alpha} u_{k}(x, t)+N\left[\widetilde{u}_{k}(x, t)\right]+L\left[\widetilde{u}_{k}(x, t)\right]-g(x, t)$.

Because $\widetilde{u}(x, t)$ is a restricted variation, we have

$$
\delta \bar{L}\left[J_{t}^{\alpha} \lambda\left(N\left[\tilde{u}_{k}(x, t)\right]+L\left[\tilde{u}_{k}(x, t)\right]-g(x, t)\right)\right]=0 .
$$

Taking the variation derivative $\delta$ on the both sides of (12), we can derive

$$
\begin{aligned}
& \delta \bar{u}_{k+1}(x, t) \\
& =\delta \bar{u}_{k}(x, t)+\delta \bar{L}\left[J _ { t } ^ { \alpha } \lambda \left(D_{t}^{\alpha} u_{k}(x, t)+N\left[\widetilde{u}_{k}(x, t)\right]\right.\right. \\
& \left.\left.+L\left[\tilde{u}_{k}(x, t)\right]-g(x, t)\right)\right] \\
& =\left(1+\bar{\lambda}(s) s^{\alpha}\right) \delta \bar{u}_{k}(x, s) .
\end{aligned}
$$

If setting the coefficient of $\delta \bar{u}_{k}(x, s)$ to zero, we can get

$$
\bar{\lambda}(s)=-\frac{1}{s^{\alpha}},
$$

and the Lagrange multiplier can be identified by using the inverse Laplace transform

$$
\lambda(t, \tau)=-\frac{(t-\tau)^{\alpha-1}}{\Gamma(\alpha)}=\frac{(-1)^{\alpha}(\tau-t)^{\alpha-1}}{\Gamma(\alpha)} .
$$

Substituting (16) into (12) and using the definition of Riemann-Liouville fractional integral operator, we get the iteration formula as follows:

$$
\begin{array}{r}
u_{k+1}(x, t)=u_{k}(x, t)-J_{t}^{\alpha}\left(D_{t}^{\alpha} u_{k}(x, t)+N\left[u_{k}(x, t)\right]\right. \\
\left.+L\left[u_{k}(x, t)\right]-g(x, t)\right) .
\end{array}
$$

\section{Adomian's Decomposition Method}

Applying the operator $J^{\alpha}$ and the inverse of the operator $D_{t}^{\alpha}$ to both sides of (1) yields

$$
\begin{aligned}
u(x, t)= & \sum_{k=0}^{m-1} \frac{\partial^{k} u}{\partial t^{k}}\left(x, 0^{+}\right) \frac{t^{k}}{k !}+J^{\alpha} g(x, t) \\
& -J^{\alpha}[L u(x, t)+N u(x, t)] .
\end{aligned}
$$

The Adomian's decomposition method [52-55] suggests that the solution $u(x, t)$ should be decomposed into the infinite series of components as

$$
u(x, t)=\sum_{n=0}^{\infty} u_{n}(x, t)
$$

and the nonlinear function in (1) be decomposed as follows:

$$
N u=\sum_{n=0}^{\infty} A_{n}
$$

where $A_{n}$ are the so-called the Adomian's polynomials.

Substituting the decomposition series equations (19) and (20) into both sides of (18) gives

$$
\begin{aligned}
\sum_{n=0}^{\infty} u_{n}(x, t)= & \sum_{k=0}^{m-1} \frac{\partial^{k} u}{\partial t^{k}}\left(x, 0^{+}\right) \frac{t^{k}}{k !}+J^{\alpha} g(x, t) \\
& -J^{\alpha}\left[L\left(\sum_{n=0}^{\infty} u_{n}(x, t)\right)+\sum_{n=0}^{\infty} A_{n}\right] .
\end{aligned}
$$


From this equation, the iterates are determined by the following recursive way:

$$
\begin{aligned}
& u_{0}(x, t)=\sum_{k=0}^{m-1} \frac{\partial^{k} u}{\partial t^{k}}\left(x, 0^{+}\right) \frac{t^{k}}{k !}+J^{\alpha} g(x, t), \\
& u_{1}(x, t)=-J^{\alpha}\left(L u_{0}+A_{0}\right), \\
& u_{2}(x, t)=-J^{\alpha}\left(L u_{1}+A_{1}\right), \\
& \vdots \\
& u_{n+1}(x, t)=-J^{\alpha}\left(L u_{n}+A_{n}\right) .
\end{aligned}
$$

The Adomian's polynomial $A_{n}$ can be calculated for all forms of nonlinearity according to specific algorithms constructed by Adomian [54]. The Adomian polynomials can be easily calculated by the homotopy perturbation method (for more details see [56]). The general formulation for an Adomian's polynomials is

$$
A_{n}=\frac{1}{n !}\left[\frac{d^{n}}{d \lambda^{n}} N\left(\sum_{n=0}^{\infty} \lambda^{k} u_{k}\right)\right]_{\lambda=0}
$$

This formula is easy to compute by using mathematical software or by writing a computer code to get as many polynomials as we need in the calculation of the numerical as well as explicit solutions. Finally, we approximate the solution $u(x, t)$ by the truncated series as

$$
\phi_{N}(x, t)=\sum_{n=0}^{N-1} u_{n}(x, t), \quad \lim _{N \rightarrow \infty} \phi_{N}(x, t)=u(x, t) .
$$

\section{Applications and Results}

From (17) and according to Lemma 4, we could get an approximate solution as

$$
\begin{aligned}
u(x, t)= & \lim _{k \rightarrow \infty} u_{k}(x, t) \\
= & \sum_{i=0}^{m-1} \frac{\partial^{i} u}{\partial t^{i}}\left(x, 0^{+}\right) \frac{t^{i}}{i !} \\
& -J_{t}^{\alpha}(N[u(x, t)]+L[u(x, t)])+J_{t}^{\alpha} g(x, t) .
\end{aligned}
$$

From (21), we could get an approximate solution as

$$
\begin{aligned}
u(x, t)= & \sum_{k=0}^{\infty} \widehat{u}_{k}(x, t) \\
= & \sum_{i=0}^{m-1} \frac{\partial^{i} u}{\partial t^{i}}\left(x, 0^{+}\right) \frac{t^{i}}{i !} \\
& -J_{t}^{\alpha}\left[\sum_{k=0}^{\infty} A_{k}+L\left(\sum_{k=0}^{\infty} \widehat{u}_{k}(x, t)\right)\right]+J_{t}^{\alpha} g(x, t),
\end{aligned}
$$

where $u(x, t)=\sum_{k=0}^{\infty} \widehat{u}_{k}(x, t)$ and $N[u(x, t)]=\sum_{n=0}^{\infty} A_{n}$.
When $\hat{u}_{k}(x, t)=u_{k}(x, t)-u_{k-1}(x, t)$ and $A_{k}=$ $N\left(\hat{u}_{k}(x, t)\right)=N\left(u_{k}(x, t)-u_{k}(x, t)\right)$, we could find that (25) and (26) are identical.

In this section, we will provide three examples for performing comparative studies. The exact solutions of these examples are known for the special cases $\alpha=1$ or 2 and have been solved in $[11,12,57]$ by using the VIM, HPM, ADM, and some other methods. It is to be noted that Lagrange multiplier of VIM in $[11,12]$ is an approximation.

Example 5. Consider the following linear time-fractional diffusion equation:

$$
\frac{\partial^{\alpha} u}{\partial t^{\alpha}}=\frac{\partial^{2} u}{\partial x^{2}}, \quad t>0, x \in R, 0<\alpha \leq 1,
$$

subject to the initial condition

$$
u(x, 0)=\sin x
$$

Momani and Odibat [11, 12, 57] have made a study about this equation by using the VIM, HPM, and the ADM and drew a conclusion that using the modified HPM is the same as the fourth-order term of the VIM solution. When $\alpha=1$, the VIM solution and the decomposition solution are identical.

By using the VIM described in [6], the iteration formula for (27) is given by

$$
u_{k+1}(x, t)=u_{k}(x, t)-\int_{0}^{t}\left(\frac{\partial^{\alpha}}{\partial \xi^{\alpha}} u_{k}(x, \xi)-\frac{\partial^{2}}{\partial x^{2}} u_{k}(x, \xi)\right) d \xi
$$

By employing the above variational iteration formula and beginning with $u_{0}=\sin x$, we can obtain the following approximations:

$$
\begin{aligned}
u_{0}(x, t) & =\sin (x), \\
u_{1}(x, t) & =(1-t) \sin (x), \\
u_{2}(x, t) & =\left(1+\frac{2 t^{2-\alpha}}{\Gamma(3-\alpha)}-2 t+\frac{1}{2} t^{2}\right) \sin (x), \\
u_{3}(x, t) & =\left(1-\frac{t^{3-2 \alpha}}{\Gamma(4-2 \alpha)}+\frac{3 t^{2-\alpha}}{\Gamma(3-\alpha)}-\frac{2 t^{3-\alpha}}{\Gamma(4-\alpha)}\right. \\
& \left.-3 t+\frac{3}{2} t^{2}-\frac{1}{6} t^{3}\right) \sin (x),
\end{aligned}
$$

and so on. The rest components of the iteration formula (29) can be obtained in the same manner.

To solve the problem with the ADM, the recurrence relation is obtained as follows:

$$
\begin{aligned}
& u_{0}(x, t)=u(x, 0)=\sin (x), \\
& u_{j+1}(x, t)=J^{\alpha}\left(L_{2 x} u_{j}(x, t)\right), \quad j \geq 0 .
\end{aligned}
$$


TABLE 1: Numerical values when $\alpha=0.5,0.75$, and 1.0 for (27).

\begin{tabular}{|c|c|c|c|c|c|c|c|c|}
\hline \multirow{2}{*}{$t$} & \multirow{2}{*}{$x$} & \multicolumn{2}{|c|}{$\alpha=0.50$} & \multicolumn{2}{|c|}{$\alpha=0.75$} & \multicolumn{3}{|c|}{$\alpha=1.0$} \\
\hline & & FVIM & VIM & FVIM & VIM & FVIM & VIM & Exact \\
\hline \multirow{4}{*}{0.25} & 0.25 & 0.14640842 & 0.14180807 & 0.17120511 & 0.16836254 & 0.19264006 & 0.19264006 & 0.19267840 \\
\hline & 0.50 & 0.28371388 & 0.27479919 & 0.33176551 & 0.32625710 & 0.37330270 & 0.37330270 & 0.37337698 \\
\hline & 0.75 & 0.40337938 & 0.39070464 & 0.47169834 & 0.46386658 & 0.53075518 & 0.53075518 & 0.53086080 \\
\hline & 1.00 & 0.49796471 & 0.48231796 & 0.58230325 & 0.57263509 & 0.65520788 & 0.65520788 & 0.65533826 \\
\hline \multirow{4}{*}{0.50} & 0.25 & 0.10790621 & 0.10407459 & 0.13273945 & 0.13274483 & 0.14947323 & 0.14947323 & 0.15005809 \\
\hline & 0.50 & 0.20910333 & 0.20167832 & 0.25722580 & 0.25723624 & 0.28965293 & 0.28965293 & 0.29078629 \\
\hline & 0.75 & 0.29729942 & 0.28674267 & 0.36571910 & 0.36573394 & 0.41182342 & 0.41182342 & 0.41343481 \\
\hline & 1.00 & 0.36701087 & 0.35397875 & 0.45147375 & 0.45149207 & 0.50838872 & 0.50838872 & 0.51037795 \\
\hline \multirow{4}{*}{0.75} & 0.25 & 0.07031034 & 0.10263146 & 0.10053521 & 0.11682841 & 0.11403776 & 0.11403776 & 0.11686536 \\
\hline & 0.50 & 0.13624913 & 0.19888179 & 0.19481962 & 0.22639299 & 0.22098521 & 0.22098521 & 0.22646459 \\
\hline & 0.75 & 0.19371660 & 0.28276661 & 0.27699110 & 0.32188155 & 0.31419287 & 0.31419287 & 0.32198335 \\
\hline & 1.00 & 0.23913971 & 0.34907038 & 0.34194061 & 0.39735708 & 0.38786553 & 0.38786553 & 0.39748275 \\
\hline
\end{tabular}

In view of (31), the first few components of the decomposition series are derived as follows:

$$
\begin{aligned}
& u_{0}(x, t)=\sin (x), \\
& u_{1}(x, t)=J^{\alpha}\left(L_{2 x} u_{0}(x, t)\right)=\frac{-t^{\alpha}}{\Gamma(\alpha+1)} \sin (x), \\
& u_{2}(x, t)=J^{\alpha}\left(L_{2 x} u_{1}(x, t)\right)=\frac{t^{2 \alpha}}{\Gamma(2 \alpha+1)} \sin (x), \\
& u_{3}(x, t)=J^{\alpha}\left(L_{2 x} u_{2}(x, t)\right)=\frac{-t^{3 \alpha}}{\Gamma(3 \alpha+1)} \sin (x),
\end{aligned}
$$

and so on. The rest of components of the decomposition series can be obtained in this manner.

The solution in series form is given by

$$
\begin{aligned}
u(x, t)= & \sin (x)-\frac{t^{\alpha}}{\Gamma(\alpha+1)} \sin (x)+\frac{t^{2 \alpha}}{\Gamma(2 \alpha+1)} \sin (x) \\
& +\cdots=\sum_{n=0}^{\infty} \frac{(-1)^{n} t^{n \alpha}}{\Gamma(n \alpha+1)} \sin (x) .
\end{aligned}
$$

To solve (27) by means of FVIM, we construct a correctional functional that reads as

$$
u_{k+1}=u_{k}(x, 0)+J^{\alpha} \frac{\partial^{2} u_{k}}{\partial x^{2}}
$$

From the initial value, we can derive

$u_{0}(x, t)=\sin (x)$,

$u_{1}(x, t)=\sin (x)\left(1-\frac{t^{\alpha}}{\Gamma(\alpha+1)}\right)$

$u_{2}(x, t)=\sin (x)\left(1-\frac{t^{\alpha}}{\Gamma(\alpha+1)}+\frac{t^{2 \alpha}}{\Gamma(2 \alpha+1)}\right)$,

$u_{3}(x, t)=\sin (x)\left(1-\frac{t^{\alpha}}{\Gamma(\alpha+1)}+\frac{t^{2 \alpha}}{\Gamma(2 \alpha+1)}-\frac{t^{3 \alpha}}{\Gamma(3 \alpha+1)}\right)$,

Consequently, the exact solution can be obtained as

$$
u(t)=\lim _{n \rightarrow \infty} u_{n}(t)=\sum_{n=0}^{\infty} \frac{(-1)^{n} t^{n \alpha}}{\Gamma(n \alpha+1)} \sin (x),
$$

which is the same as that obtained by ADM.

Table 1 shows the approximate solutions for (27) obtained for different values of $\alpha$ using methods VIM, ADM, and FVIM. The values of $\alpha=1$ are the only case for which we know the exact solution. From (33) and (36), it is obvious that the solution of (27) obtained using the FVIM is the same as the ADM. Moreover, when $\alpha$ is a positive integer, the Lagrange multiplier of FVIM is identical to that of VIM, so the solutions obtained by the two methods are the same. It should be noted that only the fourth-order term of the VIM and FVIM is used in evaluating the approximate solutions.

Example 6. We next consider the following linear time-fractional wave equation:

$$
\frac{\partial^{\alpha} u}{\partial t^{\alpha}}=\frac{1}{2} x^{2} \frac{\partial^{2} u}{\partial x^{2}}, \quad t>0, x \in R, 1<\alpha \leq 2,
$$


TABLE 2: Numerical values when $\alpha=1.5,1.75$, and 2.0 for (37).

\begin{tabular}{|c|c|c|c|c|c|c|c|c|}
\hline \multirow{2}{*}{$t$} & \multirow{2}{*}{$x$} & \multicolumn{2}{|c|}{$\alpha=1.5$} & \multicolumn{2}{|c|}{$\alpha=1.75$} & \multicolumn{3}{|c|}{$\alpha=2.0$} \\
\hline & & FVIM & VIM & FVIM & VIM & FVIM & VIM & Exact \\
\hline \multirow{4}{*}{0.25} & 0.25 & 0.26622298 & 0.26599883 & 0.26593959 & 0.26590628 & 0.26578827 & 0.26578827 & 0.26578827 \\
\hline & 0.50 & 0.56489190 & 0.56399533 & 0.56375836 & 0.56362512 & 0.56315308 & 0.56315308 & 0.56315308 \\
\hline & 0.75 & 0.89600678 & 0.89398950 & 0.89345630 & 0.89315652 & 0.89209443 & 0.89209443 & 0.89209443 \\
\hline & 1.00 & 1.25956762 & 1.25598133 & 1.25503343 & 1.25450047 & 1.25261232 & 1.25261232 & 1.25261232 \\
\hline \multirow{4}{*}{0.50} & 0.25 & 0.28474208 & 0.28393355 & 0.28340402 & 0.28328354 & 0.28256846 & 0.28256846 & 0.28256846 \\
\hline & 0.50 & 0.63896831 & 0.63573419 & 0.63361610 & 0.63313417 & 0.63027383 & 0.63027383 & 0.63027383 \\
\hline & 0.75 & 1.06267869 & 1.05540192 & 1.05063622 & 1.04955189 & 1.04311611 & 1.04311611 & 1.04311611 \\
\hline & 1.00 & 1.55587323 & 1.54293675 & 1.53446439 & 1.53253670 & 1.52109530 & 1.52109530 & 1.52109531 \\
\hline \multirow{4}{*}{0.75} & 0.25 & 0.30690489 & 0.30527637 & 0.30361709 & 0.30335993 & 0.30139478 & 0.30139478 & 0.30139480 \\
\hline & 0.50 & 0.72761955 & 0.72110549 & 0.71446834 & 0.71343972 & 0.70557913 & 0.70557913 & 0.70557918 \\
\hline & 0.75 & 1.26214400 & 1.24748736 & 1.23255378 & 1.23023936 & 1.21255304 & 1.21255304 & 1.21255316 \\
\hline & 1.00 & 1.91047821 & 1.88442198 & 1.85787338 & 1.85375886 & 1.82231652 & 1.82231652 & 1.82231673 \\
\hline
\end{tabular}

subject to the initial conditions

$$
u(x, 0)=x, \quad \frac{\partial u(x, 0)}{\partial t}=x^{2} .
$$

By using the VIM described in [6], the iteration formula for (37) is given by

$$
u_{k+1}(x, t)=u_{k}(x, t)-\int_{0}^{t}\left(\frac{\partial^{\alpha}}{\partial \xi^{\alpha}} u_{k}(x, \xi)-\frac{1}{2} x^{2} \frac{\partial^{2}}{\partial x^{2}} u_{k}(x, \xi)\right) d \xi
$$

By using variational iteration formula and beginning with $u_{0}=x+x^{2} t$, we can obtain the following approximations:

$$
\begin{gathered}
u_{1}=x+x^{2}\left(t+\frac{t^{3}}{3 !}\right), \\
u_{2}=x+x^{2}\left(t+\frac{t^{3}}{3}+\frac{t^{5}}{5 !}-\frac{t^{5-\alpha}}{\Gamma(6-\alpha)}\right), \\
u_{3}=x+x^{2}\left(t+\frac{t^{3}}{2}+\frac{t^{5}}{40}+\frac{t^{7}}{7 !}-\frac{3 t^{5-\alpha}}{\Gamma(6-\alpha)}-\frac{2 t^{7-\alpha}}{\Gamma(8-\alpha)}\right. \\
\left.+\frac{t^{7-2 \alpha}}{\Gamma(8-2 \alpha)}\right),
\end{gathered}
$$

To solve the problem by using the decomposition method, we substitute (37) and the initial conditions equation (38) into (22), and we obtain the following recurrence relation:

$$
\begin{aligned}
& u_{0}(x, t)=u(x, 0)=x+x^{2} t, \\
& u_{j+1}(x, t)=\frac{1}{2} J^{\alpha}\left(x^{2} L_{2 x} u_{j}(x, t)\right), \quad j \geq 0 .
\end{aligned}
$$

In view of (22), the first few components of the decomposition series are derived as follows:

$$
\begin{aligned}
& u_{0}(x, t)=x+x^{2} t \\
& u_{1}(x, t)=\frac{1}{2} J^{\alpha}\left(x^{2} L_{2 x} u_{0}(x, t)\right)=x^{2} \frac{t^{\alpha+1}}{\Gamma(\alpha+2)}, \\
& u_{2}(x, t)=\frac{1}{2} J^{\alpha}\left(x^{2} L_{2 x} u_{1}(x, t)\right)=x^{2} \frac{t^{2 \alpha+1}}{\Gamma(2 \alpha+2)}, \\
& u_{3}(x, t)=\frac{1}{2} J^{\alpha}\left(x^{2} L_{2 x} u_{2}(x, t)\right)=x^{2} \frac{t^{3 \alpha+1}}{\Gamma(3 \alpha+2)} .
\end{aligned}
$$

By using fractional variational iteration formula (17) and beginning with $u_{0}=x+x^{2} t$, we can obtain the following approximations:

$$
\begin{aligned}
& u_{0}=x+x^{2} t \\
& u_{1}=x+x^{2} t+\frac{1}{\Gamma(2+\alpha)} x^{2} t^{1+\alpha} \\
& u_{2}=x+x^{2}\left[t+\frac{t^{1+\alpha}}{\Gamma(2+\alpha)}+\frac{t^{1+2 \alpha}}{\Gamma(2+2 \alpha)}\right], \\
& u_{3}=x+x^{2}\left[t+\frac{t^{1+\alpha}}{\Gamma(2+\alpha)}+\frac{t^{1+2 \alpha}}{\Gamma(2+2 \alpha)}+\frac{t^{1+3 \alpha}}{\Gamma(2+3 \alpha)}\right], \\
& u_{4}=x+x^{2} \\
& x\left[t+\frac{t^{1+\alpha}}{\Gamma(2+\alpha)}+\frac{t^{1+2 \alpha}}{\Gamma(2+2 \alpha)}+\frac{t^{1+3 \alpha}}{\Gamma(2+3 \alpha)}+\frac{t^{1+4 \alpha}}{\Gamma(2+4 \alpha)}\right],
\end{aligned}
$$

Table 2 shows the approximate solutions for (37) obtained for different values of $\alpha$ using methods VIM, ADM, and FVIM. 
The values of $\alpha=2$ are the only case for which we know the exact solution $u(x, t)=x+x^{2} \sinh (t)$. From (42) and (43), it is obvious that the solution of (37) obtained by using the FVIM is the same as the ADM. As the previous example, the fourth-order term of the VIM/FVIM is utilized in evaluating the approximate solutions.

Example 7. Consider the following nonlinear time-fractional advection partial differential equation [57]:

$$
\begin{array}{r}
D_{t}^{\alpha} u(x, t)+u(x, t) u_{x}(x, t)=x+x t^{2}, \\
t>0, \quad x \in R, \quad 0<\alpha \leq 1,
\end{array}
$$

subject to the initial conditions

$$
u(x, 0)=0 \text {. }
$$

By using the VIM described in [6], the iteration formula for (44) is given by

$$
\begin{gathered}
u_{k+1}(x, t)=u_{k}(x, t)-\int_{0}^{t}\left(\frac{\partial^{\alpha}}{\partial \xi^{\alpha}} u_{k}(x, \xi)+u_{k}(x, \xi) \frac{\partial u_{k}(x, \xi)}{\partial x}\right. \\
\left.-\left(x+x \xi^{2}\right)\right) d \xi
\end{gathered}
$$

By the variational iteration method, starting with $u_{0}(x, t)=0$, we can obtain the following approximations:

$u_{0}(x, t)=0$,

$u_{1}(x, t)=x\left(t+\frac{t^{3}}{3}\right)$

$u_{2}(x, t)=x\left(2 t+\frac{t^{3}}{3}-\frac{2 t^{5}}{15}-\frac{t^{7}}{63}-\frac{t^{2-\alpha}}{\Gamma(3-\alpha)}-\frac{\Gamma(4) t^{4-\alpha}}{3 \Gamma(5-\alpha)}\right)$

In the same manner, the rest of components of the iteration formula (46) can be obtained by using the Mathematica package.

To solve the problem using the decomposition method, we obtain the following recurrence relation:

$$
\begin{gathered}
u_{0}(x, t)=u(x, 0)+J^{\alpha}\left(x+x t^{2}\right)=x\left(\frac{t^{\alpha}}{\Gamma(\alpha+1)}+\frac{2 t^{\alpha+2}}{\Gamma(\alpha+3)}\right), \\
u_{j+1}(x, t)=-J^{\alpha}\left(A_{j}\right), \quad j \geq 0,
\end{gathered}
$$

where $A_{j}$ are the Adomian's polynomials for the nonlinear function $N=u u_{x}$. In view of (22), the first few components of the decomposition series are derived as follows:

$$
\begin{gathered}
u_{0}(x, t)=x\left(\frac{t^{\alpha}}{\Gamma(1+\alpha)}+\frac{2 t^{2+\alpha}}{\Gamma(3+\alpha)}\right), \\
u_{1}(x, t)=-x\left(\frac{\Gamma(1+2 \alpha) t^{3 \alpha}}{\Gamma(1+\alpha)^{2} \Gamma(1+3 \alpha)}+\frac{4 \Gamma(3+2 \alpha) t^{3 \alpha+2}}{\Gamma(1+\alpha) \Gamma(3+\alpha) \Gamma(3+3 \alpha)}\right. \\
\left.+\frac{4 \Gamma(5+2 \alpha) t^{3 \alpha+4}}{\Gamma(3+\alpha)^{2} \Gamma(5+3 \alpha)}\right),
\end{gathered}
$$$$
u_{2}(x, t)=2 x\left(\frac{\Gamma(1+2 \alpha) \Gamma(1+4 \alpha) t^{5 \alpha}}{\Gamma(1+\alpha)^{3} \Gamma(1+3 \alpha) \Gamma(1+5 \alpha)}\right.
$$$$
\left.+\frac{8 \Gamma(5+2 \alpha)^{2} \Gamma(8+6 \alpha) t^{5 \alpha+6}}{\Gamma(3+\alpha)^{3} \Gamma(5+3 \alpha) \Gamma(7+5 \alpha)}+\cdots\right),
$$

The first three terms of the decomposition series (48) are given by

$$
\begin{gathered}
u(x, t)=x\left(\frac{t^{\alpha}}{\Gamma(1+\alpha)}+\frac{2 t^{2+\alpha}}{\Gamma(3+\alpha)}-\frac{\Gamma(1+2 \alpha) t^{3 \alpha}}{\Gamma(1+\alpha)^{2} \Gamma(1+3 \alpha)}\right. \\
\left.-\frac{4 \Gamma(3+2 \alpha) t^{3 \alpha+2}}{\Gamma(1+\alpha) \Gamma(3+\alpha) \Gamma(3+3 \alpha)}+\cdots\right) .
\end{gathered}
$$

By the FVIM and beginning with $u_{0}(x, t)=0$, we can obtain the following approximations:

$$
\begin{aligned}
& u_{k+1}(x, t)=u_{k}(x, 0)+J^{\alpha}\left(x+x t^{2}\right)-J^{\alpha}\left(u_{k} \frac{\partial u_{k}}{\partial x}\right) \\
& u_{0}(x, t)=0 \\
& u_{1}(x, t)=x\left(\frac{t^{\alpha}}{\Gamma(1+\alpha)}+\frac{2 t^{2+\alpha}}{\Gamma(3+\alpha)}\right) \\
& u_{2}(x, t) \\
& =x\left(\frac{t^{\alpha}}{\Gamma(1+\alpha)}+\frac{2 t^{2+\alpha}}{\Gamma(3+\alpha)}-\frac{\Gamma(1+2 \alpha) t^{3 \alpha}}{\Gamma(1+\alpha)^{2} \Gamma(1+3 \alpha)}\right. \\
& \quad-\frac{4 \Gamma(3+2 \alpha) t^{3 \alpha+2}}{\Gamma(1+\alpha) \Gamma(3+\alpha) \Gamma(3+3 \alpha)} \\
& \left.\quad-\frac{4 \Gamma(5+2 \alpha) t^{3 \alpha+4}}{\Gamma(3+\alpha)^{2} \Gamma(5+3 \alpha)}\right)
\end{aligned}
$$


TABLE 3: Numerical values when $\alpha=0.5,0.75$, and 1.0 for (44).

\begin{tabular}{|c|c|c|c|c|c|c|c|c|}
\hline \multirow{2}{*}{$t$} & \multirow{2}{*}{$x$} & \multicolumn{2}{|c|}{$\alpha=0.5$} & \multicolumn{2}{|c|}{$\alpha=0.75$} & \multicolumn{3}{|c|}{$\alpha=1.0$} \\
\hline & & FVIM & VIM & FVIM & VIM & FVIM & VIM & Exact \\
\hline \multirow{4}{*}{0.25} & 0.25 & 0.12422501 & 0.12306887 & 0.09230374 & 0.09291265 & 0.06250058 & 0.06250058 & 0.062500 \\
\hline & 0.50 & 0.24845002 & 0.24613773 & 0.18460748 & 0.18582531 & 0.12500117 & 0.12500117 & 0.125000 \\
\hline & 0.75 & 0.37267504 & 0.36920660 & 0.27691122 & 0.27873796 & 0.18750175 & 0.18750175 & 0.187500 \\
\hline & 1.00 & 0.49690005 & 0.49227547 & 0.36921496 & 0.37165062 & 0.25000234 & 0.25000234 & 0.250000 \\
\hline \multirow{4}{*}{0.50} & 0.25 & 0.18377520 & 0.19472445 & 0.15148283 & 0.15611713 & 0.12507592 & 0.12507592 & 0.125000 \\
\hline & 0.50 & 0.36755040 & 0.38944890 & 0.30296566 & 0.31223426 & 0.25015184 & 0.25015184 & 0.250000 \\
\hline & 0.75 & 0.55132559 & 0.58417334 & 0.45444848 & 0.46835139 & 0.37522776 & 0.37522776 & 0.375000 \\
\hline & 1.00 & 0.73510079 & 0.77889779 & 0.60593131 & 0.62446853 & 0.50030368 & 0.50030368 & 0.500000 \\
\hline \multirow{4}{*}{0.75} & 0.25 & 0.27227270 & 0.22829012 & 0.21407798 & 0.20170432 & 0.18881843 & 0.18881843 & 0.187500 \\
\hline & 0.50 & 0.54454540 & 0.45658025 & 0.42815596 & 0.40340864 & 0.37763687 & 0.37763687 & 0.375000 \\
\hline & 0.75 & 0.81681810 & 0.68487037 & 0.64223394 & 0.60511296 & 0.56645530 & 0.56645530 & 0.562500 \\
\hline & 1.00 & 1.08909080 & 0.91316050 & 0.85631192 & 0.80681728 & 0.75527373 & 0.75527373 & 0.750000 \\
\hline
\end{tabular}

$$
\begin{aligned}
& u_{3}(x, t) \\
& =x\left(\frac{t^{\alpha}}{\Gamma(1+\alpha)}+\frac{2 t^{2+\alpha}}{\Gamma(3+\alpha)}-\frac{\Gamma(1+2 \alpha) t^{3 \alpha}}{\Gamma(1+\alpha)^{2} \Gamma(1+3 \alpha)}\right. \\
& -\frac{4 \Gamma(3+2 \alpha) t^{3 \alpha+2}}{\Gamma(1+\alpha) \Gamma(3+\alpha) \Gamma(3+3 \alpha)} \\
& -\frac{4 \Gamma(5+2 \alpha) t^{3 \alpha+4}}{\Gamma(3+\alpha)^{2} \Gamma(5+3 \alpha)} \\
& +2\left(\frac{\Gamma(1+2 \alpha) \Gamma(1+4 \alpha) t^{5 \alpha}}{\Gamma(1+\alpha)^{3} \Gamma(1+3 \alpha) \Gamma(1+5 \alpha)}\right. \\
& \left.\left.+\frac{8 \Gamma(5+2 \alpha)^{2} \Gamma(8+6 \alpha) t^{5 \alpha+6}}{\Gamma(3+\alpha)^{3} \Gamma(5+3 \alpha) \Gamma(7+5 \alpha)}+\cdots\right)\right) .
\end{aligned}
$$

Table 3 shows the approximate solutions for (44) obtained for different values of $\alpha$ using methods VIM, ADM, and FVIM. The values of $\alpha=1$ is the only case for which we know the exact solution $u(x, t)=x t$. From (49) and (52), it is obvious that the solution of (44) obtained using the FVIM is the same as that of ADM. As the previous examples, the fourth-order term of the VIM solution and four terms of the FVIM are used in evaluating the approximate solutions for Table 3.

\section{Conclusion}

The main goal of this work is to conduct a comparative study between fractional variational iteration method and the Adomian's decomposition method. The two methods are powerful and effective tools for the solution of fractional partial differential equations, and both give approximations of higher accuracy and closed form solutions if existing. There are three important points to make here. First, FVIM is identical to the decomposition method in some sense. Second,
FVIM reduces the computational workload by avoiding the evaluation of Adomian's polynomials, hence the iteration is straightforward. Third, FVIM provides the components of the exact solution like the ADM, but there is no need to add successive components to get the series solution. So, FVIM is more effective than $\mathrm{ADM}$ in solving the fractional partial differential equations.

\section{Acknowledgments}

This work is supported by National Natural Science Foundation of China (Grant no. 41105063). The authors are very grateful to reviewers for carefully reading the paper and for his (her) comments and suggestions which have improved the paper.

\section{References}

[1] R. P. Agarwal, B. de Andrade, and C. Cuevas, "Weighted pseudo-almost periodic solutions of a class of semilinear fractional differential equations," Nonlinear Analysis: Real World Applications, vol. 11, no. 5, pp. 3532-3554, 2010.

[2] R. P. Agarwal, V. Lakshmikantham, and J. J. Nieto, "On the concept of solution for fractional differential equations with uncertainty," Nonlinear Analysis: Theory, Methods \& Applications, vol. 72, no. 6, pp. 2859-2862, 2010.

[3] R. Garrappa and M. Popolizio, "On the use of matrix functions for fractional partial differential equations," Mathematics and Computers in Simulation, vol. 81, no. 5, pp. 1045-1056, 2011.

[4] R. Hilfer, Applications of Fractional Calculus in Physics, World Scientific Publishing, River Edge, NJ, USA, 2000.

[5] Y. Khan, N. Faraz, A. Yildirim, and Q. Wu, "Fractional variational iteration method for fractional initial-boundary value problems arising in the application of nonlinear science," Computers \& Mathematics with Applications, vol. 62, no. 5, pp. 2273-2278, 2011.

[6] Z. Odibat, "On Legendre polynomial approximation with the VIM or HAM for numerical treatment of nonlinear fractional differential equations," Journal of Computational and Applied Mathematics, vol. 235, no. 9, pp. 2956-2968, 2011. 
[7] I. Petráš, "A note on the fractional-order Chua's system," Chaos, Solitons \& Fractals, vol. 38, no. 1, pp. 140-147, 2008.

[8] I. Podlubny, Fractional Differential Equations, vol. 198 of Mathematics in Science and Engineering, Academic Pres, San Diego, Calif, USA, 1999.

[9] S. G. Samko, A. A. Kilbas, and O. I. Marichev, Fractional Integrals and Derivatives: Theory and Applications, Gordon and Breach, Yverdon, Switzerland, 1993.

[10] Z. Odibat and S. Momani, "Numerical methods for nonlinear partial differential equations of fractional order," Applied Mathematical Modelling, vol. 32, no. 1, pp. 28-39, 2008.

[11] S. Momani and Z. Odibat, "Analytical approach to linear fractional partial differential equations arising in fluid mechanics," Physics Letters A, vol. 355, no. 4-5, pp. 271-279, 2006.

[12] S. Momani and Z. Odibat, "Analytical solution of a timefractional Navier-Stokes equation by Adomian decomposition method," Applied Mathematics and Computation, vol. 177, no. 2, pp. 488-494, 2006.

[13] G.-C. Wu and J.-H. He, "Fractional adomian decomposition method," http://arxiv.org/abs/1006.5264v1 .

[14] Z. Odibat and S. Momani, "Modified homotopy perturbation method: application to quadratic Riccati differential equation of fractional order," Chaos, Solitons \& Fractals, vol. 36, no. 1, pp. 167-174, 2008.

[15] S. H. Hosseinnia, A. Ranjbar, and S. Momani, "Using an enhanced homotopy perturbation method in fractional differential equations via deforming the linear part," Computers \& Mathematics with Applications, vol. 56, no. 12, pp. 3138-3149, 2008.

[16] O. Abdulaziz, I. Hashim, and S. Momani, "Solving systems of fractional differential equations by homotopy-perturbation method," Physics Letters A, vol. 372, no. 4, pp. 451-459, 2008.

[17] J. H. He, "Variational iteration method-a kind of non-linear analytical technique: some examples," International Journal of Non-Linear Mechanics, vol. 34, no. 4, pp. 699-708, 1999.

[18] J.-H. He, "Variational iteration method for autonomous ordinary differential systems," Applied Mathematics and Computation, vol. 114, no. 2-3, pp. 115-123, 2000.

[19] J.-H. He, "Variational principles for some nonlinear partial differential equations with variable coefficients," Chaos, Solitons \& Fractals, vol. 19, no. 4, pp. 847-851, 2004.

[20] J.-H. He and X.-H. Wu, "Variational iteration method: new development and applications," Computers \& Mathematics with Applications, vol. 54, no. 7-8, pp. 881-894, 2007.

[21] J.-H. He, "Variational iteration method-some recent results and new interpretations," Journal of Computational and Applied Mathematics, vol. 207, no. 1, pp. 3-17, 2007.

[22] J. H. He, G. C. Wu, and F. Austin, "The variational iterational method which should be follow," Nonlinear Science Letters A, vol. 1, no. 1, pp. 1-30, 2010.

[23] S. Liao, "Homotopy analysis method: a new analytical technique for nonlinear problems," Communications in Nonlinear Science and Numerical Simulation, vol. 2, no. 2, pp. 95-100, 1997.

[24] S. Liao, "On the homotopy analysis method for nonlinear problems," Applied Mathematics and Computation, vol. 147, no. 2, pp. 499-513, 2004.

[25] L. Song and H. Zhang, "Application of homotopy analysis method to fractional KdV-Burgers-Kuramoto equation," Physics Letters A, vol. 367, no. 1-2, pp. 88-94, 2007.

[26] Subir das'R. Kumar and P. K. Gupta, "Approximate analytical solutions for fractional space- and time-partial differential equations using homotopy analysis method," Applications and Applied Mathematics, vol. 5, no. 2, pp. 544-562, 2010.

[27] Z.-B. Li and J.-H. He, "Fractional complex transform for fractional differential equations," Mathematical \& Computational Applications, vol. 15, no. 5, pp. 970-973, 2010.

[28] Z. B. Li, “An Extended fractional complex transform," International Journal of Nonlinear Sciences and Numerical Simulation, vol. 11, pp. 335-337, 2010.

[29] J.-H. He, S. K. Elagan, and Z. B. Li, "Geometrical explanation of the fractional complex transform and derivative chain rule for fractional calculus," Physics Letters A, vol. 376, no. 4, pp. 257259, 2012.

[30] J. H. He and Z. B. Li, "Converting fractional differential equations into partial differential equations," Thermal Science, vol. 16, no. 2, pp. 331-334, 2012.

[31] Z. B. Li and W. H. Zhu, "Exact solutions of time-fractional heat conduction equations by the fractional complex transform," Thermal Science, vol. 16, no. 2, pp. 335-338, 2012.

[32] A. H. Bhrawy and M. M. Al-Shomrani, "A shifted Legendre spectral method for fractional-order multi-point boundary value problems," Advances in Difference Equations, vol. 2012, article 8, 2012.

[33] M. M. Khader and A. S. Hendy, "The approximate and exact solutions of the fractional-order delay differential equations using Legendre seudospectral Method," International Journal of Pure and Applied Mathematics, vol. 74, no. 3, pp. 287-297, 2012.

[34] H. Jafari, S. A. Yousefi, M. A. Firoozjaee, S. Momani, and C. M. Khalique, "Application of Legendre wavelets for solving fractional differential equations," Computers \& Mathematics with Applications, vol. 62, no. 3, pp. 1038-1045, 2011.

[35] J.-H. He, "Approximate analytical solution for seepage flow with fractional derivatives in porous media," Computer Methods in Applied Mechanics and Engineering, vol. 167, no. 1-2, pp. 57-68, 1998.

[36] J. H. He, "Asymptotic methods for solitary solutions and compactons," Abstract and Applied Analysis, vol. 2012, Article ID 916793, 130 pages, 2012.

[37] R. Y. Molliq, M. S. M. Noorani, and I. Hashim, "Variational iteration method for fractional heat- and wave-like equations," Nonlinear Analysis: Real World Applications, vol. 10, no. 3, pp. 1854-1869, 2009.

[38] Z. M. Odibat and S. Momani, "Application of variational iteration method to nonlinear differential equations of fractional order," International Journal of Nonlinear Sciences and Numerical Simulation, vol. 7, no. 1, pp. 27-34, 2006.

[39] G. E. Drăgănescu, "Application of a variational iteration method to linear and nonlinear viscoelastic models with fractional derivatives," Journal of Mathematical Physics, vol. 47, no. 8, p. 082902, 9, 2006.

[40] D. D. Ganji and S. H. Hashemi Kachapi, "Analysis of nonlinear equations in fluids," Progress in Nonlinear Science, vol. 2, pp. 1293, 2011.

[41] D. D. Ganji and S. H. Hashemi Kachapi, "Analytical and numerical methods in engineering and applied sciences," Progress in Nonlinear Science, vol. 3, pp. 1-579, 2011.

[42] G.-C. Wu, "A fractional variational iteration method for solving fractional nonlinear differential equations," Computers \& Mathematics with Applications, vol. 61, no. 8, pp. 2186-2190, 2011.

[43] J.-H. He, "A short remark on fractional variational iteration method," Physics Letters A, vol. 375, no. 38, pp. 3362-3364, 2011. 
[44] N. Faraz, Y. Khan, H. Jafari, A. Yildirim, and M. Madani, "Fractional variational iteration method via modified RiemannLiouville derivative," Journal of King Saud University-Science, vol. 23, pp. 413-417, 2011.

[45] J.-I. Gu and T.-C. Xia, "Handling the fractional Boussinesq-like equation by fractional variational iteration method," Communication on Applied Mathematics and Computation, vol. 25, no. 1, pp. 46-52, 2011.

[46] A.-M. Wazwaz, "A comparison between the variational iteration method and Adomian decomposition method," Journal of Computational and Applied Mathematics, vol. 207, no. 1, pp.129136, 2007.

[47] A. M. Siddiqui, A. A. Farooq, T. Haroon, and B. S. Babcock, "A comparison of variational iteration and Adomian decomposition methods in solving nonlinear thin film flow problems," Applied Mathematical Sciences, vol. 6, no. 99, pp. 4911-4919, 2012.

[48] J. Biazar, P. Gholamin, and K. Hosseini, "Variational iteration and Adomian decomposition methods for solving Kawahara and modified Kawahara equations," Applied Mathematical Sciences, vol. 2, no. 55, pp. 2705-2712, 2008.

[49] A.-M. Wazwaz and R. Rach, "Comparison of the Adomian decomposition method and the variational iteration method for solving the Lane-Emden equations of the first and second kinds," Kybernetes, vol. 40, no. 9-10, pp. 1305-1318, 2011.

[50] K. S. Miller and B. Ross, An Introduction to the Fractional Calculus and Fractional Differential Equations, John Wiley \& Sons, New York, NY, USA, 1993.

[51] Y. Luchko and R. Gorenflo, "An operational method for solving fractional differential equations with the Caputo derivatives," Acta Mathematica Vietnamica, vol. 24, no. 2, pp. 207-233, 1999.

[52] G. Adomian, "A review of the decomposition method in applied mathematics," Journal of Mathematical Analysis and Applications, vol. 135, no. 2, pp. 501-544, 1988.

[53] G. Adomian, Solving Frontier Problems of Physics: The Decomposition Method, vol. 60 of Fundamental Theories of Physics, Kluwer Academic, Boston, Mass, USA, 1994.

[54] A.-M. Wazwaz, "A new algorithm for calculating Adomian polynomials for nonlinear operators," Applied Mathematics and Computation, vol. 111, no. 1, pp. 33-51, 2000.

[55] A.-M. Wazwaz and S. M. El-Sayed, "A new modification of the Adomian decomposition method for linear and nonlinear operators," Applied Mathematics and Computation, vol. 122, no. 3, pp. 393-405, 2001.

[56] A. Ghorbani and J. Saberi-Nadjafi, "He’s homotopy perturbation method for calculating adomian polynomials," International Journal of Nonlinear Sciences and Numerical Simulation, vol. 8, no. 2, pp. 229-232, 2007.

[57] S. Momani and Z. Odibat, "A novel method for nonlinear fractional partial differential equations: combination of DTM and generalized Taylor's formula," Journal of Computational and Applied Mathematics, vol. 220, no. 1-2, pp. 85-95, 2008. 


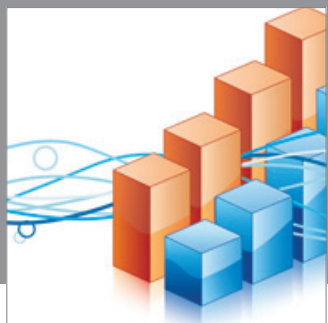

Advances in

Operations Research

mansans

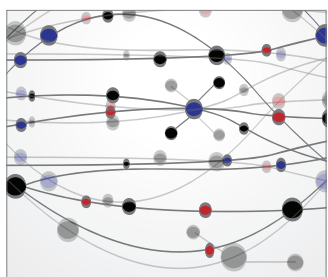

The Scientific World Journal
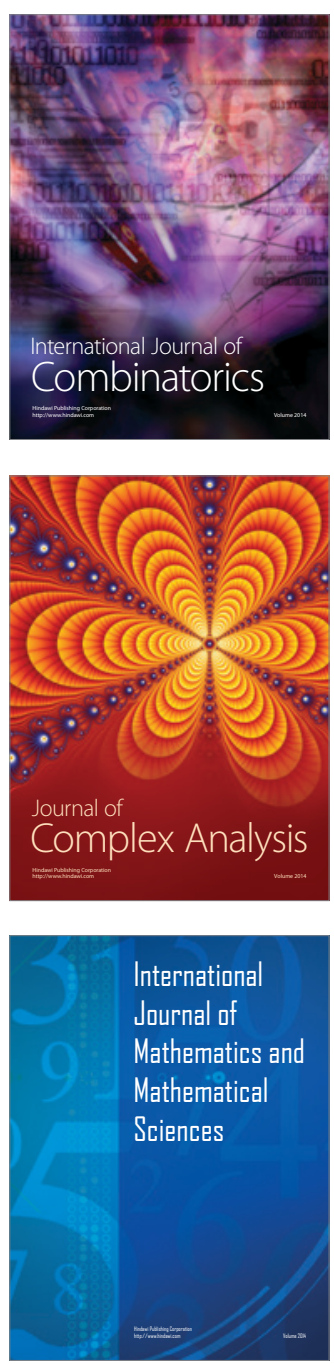
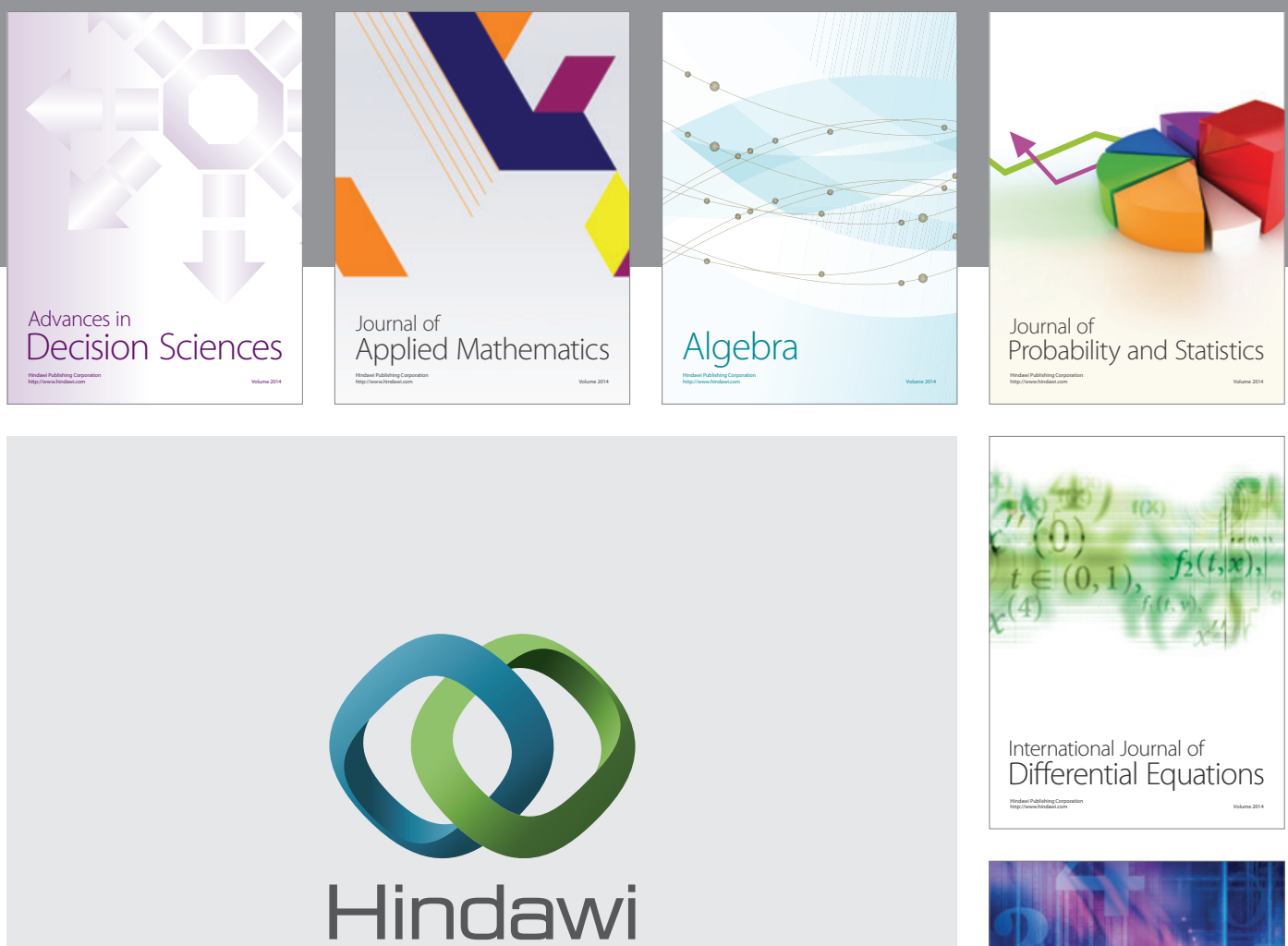

Submit your manuscripts at http://www.hindawi.com
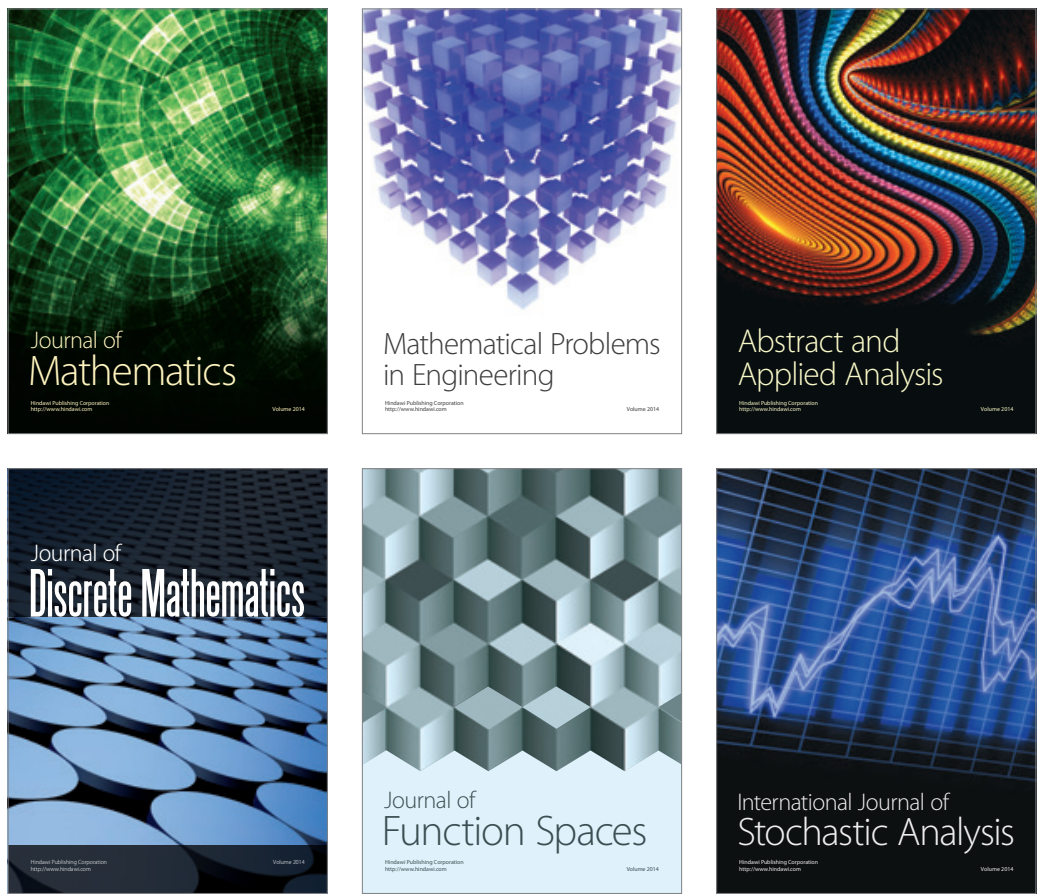

Journal of

Function Spaces

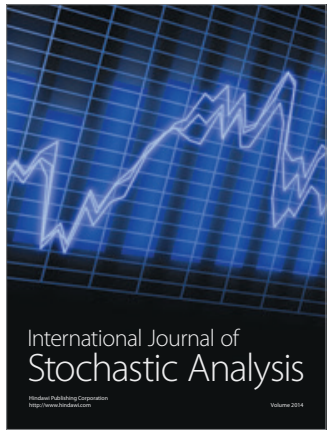

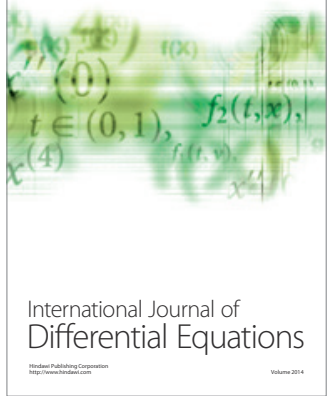
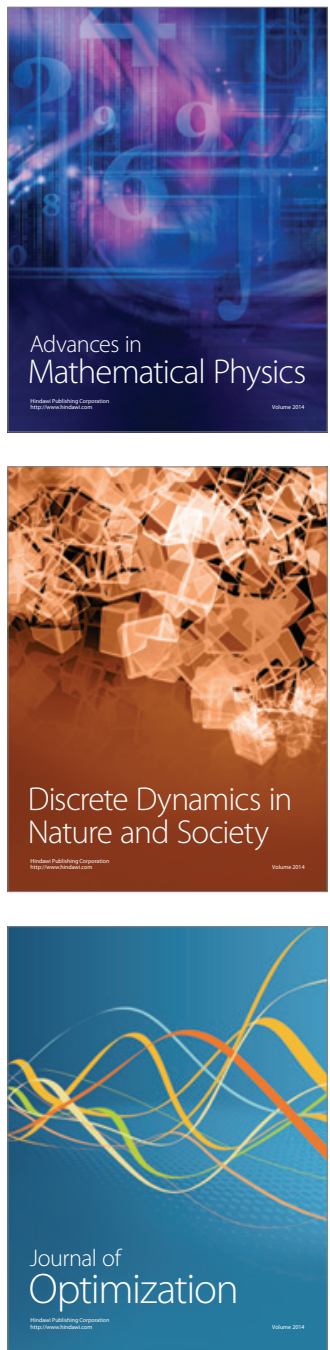